\title{
Guide pour l'entretien médical d'information
}

\section{J. Pók, P. Wyss, C. Honegger}

Pour la Commission pour l'assurance de qualité de la gynécologie suisse/ SSGO (Président: Prof. Dr Daniel Surbek)
Correspondance:

Prof. Dr Daniel Surbek

Universitäts-Frauenklinik

Inselspital

Effingerstrasse 102

CH-3010 Berne

Tél. 0316321103

Fax 0316321105

qsk-sggg@insel.ch

\section{Préambule}

L'information de nos patientes sur les pathologies, les mesures diagnostiques et thérapeutiques et en particulier les interventions chirurgicales prévues, fait partie intégrante de la prise en charge médicale. L'acceptation occidentale des concepts d'autodétermination et de droit à l'autonomie implique une information des patientes complète, compréhensible et pertinente. En dehors de ces aspects éthiques, l'information des patientes est également indispensable au plan juridique. Dans ce cadre, elle constitue une obligation pour le médecin avant que la patiente ne donne un consentement et un mandat de traitement. En cas d'information insuffisante, le consentement de la patiente est moins probant et pour le médecin traitant la justification de l'intervention devient difficile à défendre. Lorsque la patiente confie un mandat de traitement à son médecin, ce dernier ne risque pas de plainte pour lésions corporelles en raison du seul acte médical.

L'information doit être utile à la patiente dans le cadre de sa prise de décision, mais ne devrait si possible pas l'angoisser. Une information approfondie et satisfaisante pour la patiente doit permettre au médecin d'être un partenaire dans le processus de décision («shared decision making»).

\section{Préparation de l'entretien}

Il convient de clarifier un certain nombre de points pour préparer l'entretien d'information:

\section{Problèmes de langue}

Il faut s'assurer que la patiente maîtrise suffisamment notre langue ou qu'elle soit accompagnée par une personne de confiance qui parle suffisamment bien le français.

\section{Entretien d'information avec des mineurs}

Ce n'est pas l'âge qui est déterminant mais la capacité de discernement de la personne concernée par rapport à l'objet de l'information, respectivement le traitement proposé. Le médecin doit décider si, d'après son appréciation personnelle, une patiente est capable de discernement par rapport à la question spécifique qu'il va traiter avec elle. Cette décision doit être documentée par écrit. Cela est particulièrement important avant de procéder à une stérilisation (considérer la loi en vigueur). L'information d'une patiente mineure doit être documentée de façon particulièrement soigneuse pour se protéger contre une plainte pour lésions corporelles.

\section{Planification de l'entretien}

Les lois cantonales doivent être respectées (par exemple, dans le canton de Zurich, l'entretien d'information précédant une intervention importante doit avoir lieu au moins 3 jours avant). Selon les situations, il est conseillé de convier la patiente à un entretien d'information lors d'une consultation précédant l'hospitalisation ou l'opération. Idéalement, l'information concernant une opération devrait être donnée quelques jours avant l'intervention.

\section{Moyens accessoires}

Il peut être utile de disposer de moyens accessoires (par exemple, images, esquisses, brochures d'information, livres, etc.) pour illustrer la teneur des informations.

L'entretien d'information proprement dit doit être documenté au moyen des protocoles développés par gynécologie suisse/SSGO.

\section{Déroulement de I'entretien}

\section{Maladie / Plaintes}

Récapitulation des examens ayant mené au diagnostic et au traitement proposé. Ne pas oublier de proposer à la patiente de poser des questions sur les aspects qui lui paraissent peu clairs.

\section{Mesures proposées et alternatives}

Donner le nom de l'intervention et décrire les alternatives (traitements conservateurs, autres accès chirurgicaux, méthodes opératoires différentes, traitements médicamenteux ou symptomatiques). De même, il faut informer les patientes des conséquences possibles d'un refus de traitement.

\section{Indication / Exécution et extension possible de l'intervention}

Expliquer clairement pourquoi cette intervention est prévue. L'utilité et les avantages de l'intervention planifiée doivent à nouveau être mis en évidence. Ceci est valable en particulier pour 
les interventions ayant des conséquences graves (par exemple, intervention mutilante). De la même manière, l'urgence doit également être discutée.

Le déroulement de l'intervention au plan technique doit être décrit par des mots simples et illustré par des croquis. L'utilisation des moyens auxiliaires décrits plus haut est particulièrement utile dans cette phase de l'entretien.

Une extension de l'intervention peut être nécessaire en raison de la pathologie de base ou de l'apparition de complications. A ce propos, l'étendue d'une éventuelle extension doit être discutée au préalable en décrivant, le cas échéant, la possibilité d'une intervention en un ou deux temps.

Lors d'intervention à la demande de la patiente, il faut indiquer tout particulièrement les complications possibles et les désavantages éventuels.

\section{Mesures complémentaires}

Il faut mentionner la position sur la table d'opération, les voies veineuses, la prophylaxie antibiotique ou antithrombotique, l'utilisation de cathéter, de tocolyse péri-opératoire, la nécessité d'une surveillance en soins intensifs, en salle de réveil, la durée de séjour, une éventuelle préparation intestinale, etc.

\section{Risques / Conséquences / Désavantages et probabilités de succès}

Décrire les risques significatifs qui pourraient influencer la décision de la patiente. Cela comprend les risques généraux les plus fréquents (thromboses, hémorragies, infections) et les risques spécifiques de l'intervention projetée. Il faut mentionner également la possibilité d'un saignement abondant nécessitant des transfusions. La probabilité d'une transfusion de sang de donneurs et la possibilité d'une autotransfusion doivent également être mentionnées (les coûts de la préparation d'une autotransfusion sont à la charge de la patiente si le sang n'est pas utilisé). D'autres risques seront discutés si la patiente pose des questions spécifiques ou si l'anamnèse personnelle de la patiente fait craindre des risques qui d'habitude ne sont pas importants.
Les probabilités de succès doivent être clairement décrites à la patiente.

\section{Après l'intervention}

Le déroulement de la phase postopératoire doit également être discuté, et en particulier les éventuels désagréments (cathéter, nutrition, perfusion, mobilisation, etc.). La durée de l'hospitalisation et la durée de l'arrêt de travail doivent aussi être précisées pour que la patiente puisse s'organiser.

Des indications sur la période suivant immédiatement le retour à domicile sont utiles et facilitent également la planification. Il faut mentionner en particulier un éventuel traitement postopératoire, la nécessité de se ménager au plan physique, un possible séjour dans un lieu de convalescence, l'indépendance à domicile ou la nécessité d'aide. Dans ce cadre, il faut bien sûr considérer l'environnement social de la patiente.

De plus, des conseils spécifiques doivent être donnés à propos des activités de tous les jours (par exemple, sport, soins corporels, loisirs entre autres).

\section{Anesthésie}

Citer les méthodes d'anesthésie possibles pour l'intervention projetée et adresser la patiente à un anesthésiste qui, de son côté, fera un entretien d'information spécifique.

\section{Coût}

La prise en charge des coûts par la caisse-maladie doit être discutée et clarifiée. En cas d'assurance complémentaire, se renseigner sur le statut miprivé ou privé.

A la fin de l'entretien, il faut mentionner que dans tous les cas, il y a la possibilité de poser d'autres questions en cas d'information manquante.

Le protocole d'information préopératoire ou prétraitement (informed consent) sera établi avant l'intervention, signé par les deux parties et une copie sera remise à la patiente.

Réserver du temps à la fin de l'entretien pour des questions et mentionner la possibilité de se laisser un délai de réflexion. 